having pain interference (extremely, quite a bit, moderately) and having no pain interference (a little bit, not at all).

Life-Course SEP Measures (1) Age left school (young adulthood SEP): those who left school at $\leq$ the minimum school leaving age assigned low SEP; those who left school at $>$ the minimum school leaving age assigned high SEP, (2) Longest job (adult working life SEP) and (3) Current/recent job (most recent adult SEP): using the National Statistics Socio-economic Classification, Routine and manual occupations were assigned low SEP; Intermediate and Managerial and professional occupations were assigned high SEP. Life-course SEP trajectories were constructed for each respondent from the three measures.

Other Measures BMI, HADS, health locus of control, adequacy of income.

Analysis Confined to participants who provided data at three SEP time-points $(n=2535)$. Association of pain interference with each SEP trajectory (High, High, High ( $\mathrm{HHH}$ ) as reference trajectory) was calculated by logistic regression and adjusted for age, gender and BMI. Forward stepwise logistic regression was used to adjust for potential confounding psychosocial and social factors. Latent class analysis identified any clustering in SEP trajectories.

Results Adjusted response to the three stages of the survey was $71-85 \%$. The LLL SEP trajectory was significantly associated with pain interference compared to HHH (OR 2.73; 95\% CI 2.16 to 3.45 ); this association was not altered by age or gender. Adjustment for the remaining factors reduced the association but it remained significant (OR 2.05; 95\% CI 1.56 to 2.70). Latent class analysis identified two clusters of SEP trajectories: those that started Low remained Low, those starting High remained High.

Conclusion In this study, adults with a consistently low SEP throughout their life-course were more likely to report pain interference in later life.

\section{P22 CHILDHOOD SOCIOECONOMIC POSITION AND ADULT SMOKING: ARE CHILDHOOD COGNITIVE ABILITY, PSYCHOSOCIAL ADJUSTMENT AND PARENTAL INVOLVEMENT IMPORTANT?}

doi:10.1136/jech.2010.120477.22

R Lacey, N Cable, M Stafford, M Bartley, H Pikhart. Department of Epidemiology and Public Health, University College London, London, UK

Background Studies have shown that childhood socioeconomic position (SEP) is related to smoking in adulthood, independent of adult SEP. Educational attainment partially mediates this association; however, previous studies suggest three childhood factors which may also be important-childhood cognitive ability, psychosocial adjustment and parental involvement.

Objective To assess whether childhood psychosocial adjustment, cognitive ability and parental involvement are important in the association between childhood SEP and adult smoking status, over and above educational attainment.

Data, Participants and Variables Data on 7709 participants from four sweeps of the 1958 National Child Development Study were used for this study - birth (1958), age 7 (1965), age 16 (1974) and age 42 (2000). Childhood SEP was indicated using father's social class at birth and adult smoking status was taken at age 42 years (categorised as: 1 never/infrequent smokers, 2 ex-smokers, 3 current smokers). All childhood factors investigated were measured at age 7 years. Childhood cognitive ability was measured using score in the Copying Designs Test, psychosocial adjustment was measured using the teacher-assessed Bristol Social Adjustment Guide (BSAG) and parental involvement was based on questions asked of the mother and father regarding the frequency of occasions spent reading and on outings with the child. Confounding variables included were mother's smoking, age at birth of child and educational level, number of siblings and participant's own educational attainment at age 16 years (indicated by exam scores). Those with complete data on all variables used were included in the analysis.

Statistical Methods Multinomial logistic regression was used to examine the associations of childhood SEP and childhood factors with adult smoking status, both independently and mutually adjusted, and then adjusted for confounders and participant's educational attainment. Analyses were conducted separately for men and women.

Results Childhood SEP was an important predictor of current adult smoking status, even after adjustment for childhood factors and educational attainment. Also, parental involvement for men (most vs least frequent parental reading OR $0.75,95 \%$ CI 0.60 to 0.94 ; most vs least frequent parental outings OR $0.55,95 \%$ CI 0.35 to 0.86 ), and both parental involvement (most vs least frequent parental reading OR $0.68,95 \%$ CI 0.55 to 0.85 ) and psychosocial adjustment (most vs least maladjusted OR 1.28, 95\% CI 1.01 to 1.64) for women, remained important determinants of current adult smoking over and above childhood SEP, other childhood factors and educational attainment.

Conclusions These findings suggest that childhood disadvantage is associated with adult smoking behaviours and the early childhood social environment is important in the development of these.

\section{Children/Adolescence}

\section{P23 PATTERNS OF ANTIRETROVIRAL THERAPY IN A EUROPEAN STUDY OF HIV-INFECTED CHILDREN AND ADOLESCENTS}

doi:10.1136/jech.2010.120477.23

N M Alam, M Cortina-Borja, C Thorne. UCL Institute of Child Health, London, UK

Background Antiretroviral therapy (ART) has resulted in increasing median survival times in HIV-infected individuals by sustaining viral load suppression. Since children and adolescents are likely to have long-term exposure to ART, it is important to understand patterns of drug use to investigate the emergence of unintended sequelae.

Aim To investigate patterns of ART in children and adolescents Study Design Cross-sectional analysis of HIV-infected subjects aged 2-22 years across 15 clinical sites in Belgium, Italy and Poland.

Method Prevalence of both "ever-use" and "current-use" (at recruitment) of ART drug classes: nucleoside analogue reverse transcriptase inhibitors (NRTIs); non-nucleoside analogue reverse transcriptase inhibitors (NNRTIs); and protease inhibitors (PIs), and individual drugs were investigated.

Results Among 468 participants (51\% female) the median age was 13.5 years (IOR 9.9-17.0) with $320(68 \%)$ of white ethnicity and 104 (22\%) Black African. Overall, 291(62\%) were virologically suppressed (HIV-RNA $\leq 50 \mathrm{copies} / \mathrm{ml}$ ) at enrolment and $35(7 \%)$ had severe immuno-suppression (age-stratified CD4\%). Only $23(5 \%)$ subjects were ART-naive (median age $=10.0$ years, IOR; 6.1-14.3); 15 had experienced moderate or severe immuno-suppression/HIVdisease, with 15 having detectable viral load at recruitment. Of the ever-treated subjects, $98 \%(n=436)$ had received zidovudine or lamivudine in the past and $72 \%(n=324)$ currently; $82 \%(n=368)$ and $72 \% \quad(n=323)$ had been ever-exposed to PIs and NNRTIs, respectively. Median and modal lifetime number of drugs was six. Over their lifetime, $119(27 \%)$ subjects had been exposed to $\geq 8$ drugs (median age $=15.7$ years, IOR; 12.3-18.0). Age was associated with duration of total drug use $(p<0.001)$, with median age of ART initiation of 3.6 years (IOR: 1.1-7.4) Median total duration of drug use was 8.7 years ( $\mathrm{IOR}=5.7-11.2$ years). Nine percent of currently treated subjects $(\mathrm{n}=38)$ had suboptimal management, defined as NRTIs-only (24 showing evidence of incomplete viral suppression), and $91 \%(n=384)$ had treatment with combination ART (cART). The most common PI in current-use was kaletra ( $n=170,40 \%)$, 
possibly reflecting its availability as the only combination PI accessible in tablet/liquid form. Current use of PIs was associated $(p<0.001)$ with hypercholesterolaemia in adjusted analyses indicating a long-term consequence of specific ART.

Conclusions The majority of subjects had been managed with ART, with first exposure occurring at an early age. At least a quarter of participants had been treated with multiple individual drugs suggesting cumulative exposure and switching between regimens. The most prevalent treatment approach at recruitment was cART. However, there is evidence of continued use of suboptimal management strategies, and hypercholesterolaemia being associated with PI use.

\section{P24 A CLUSTER-RANDOMISED CONTROLLED TRIAL TO TEST THE EFFECTIVENESS OF A HAND WASHING INTERVENTION IN REDUCING INFECTION-RELATED ABSENCE IN PRIMARY SCHOOLS: INSIGHTS FROM AN EMBEDDED PROCESS EVALUATION}

doi:10.1136/jech.2010.120477.24

C R Chittleborough, R Campbell, A L Nicholson, S Gunn. Department of Social Medicine, University of Bristol, Bristol, UK

Objective To conduct a qualitative process evaluation within a cluster-randomised trial of an educational resource intervention to promote hand washing in primary schools and thus reduce absenteeism by reducing the transmission of respiratory and gastrointestinal infections.

Design Focus groups with pupils including drawings of hand washing facilities, semi-structured interviews with teachers, direct observation of intervention delivery and hand washing facilities.

Setting State primary schools within six local authority areas in the South West of England $(n=178)$ were randomised to receive the "Hands up for Max!" intervention in October 2009 (intervention schools) or in Autumn 2010 after all trial follow-up data are collected (control schools). Four intervention and four control schools were selected for the process evaluation from the 24 schools participating in a substudy to collect enhanced absenteeism data.

Participants Pupils in years 2 to $6(n=95)$, and key stage $1(n=8)$ and key stage $2(n=8)$ teachers.

Main Outcome Measures The process evaluation examined how the "Hands up for Max!" educational resource was delivered in intervention schools and explored responses to the intervention among pupils and staff. Ideas, attitudes, knowledge and behaviours relating to hand hygiene and hand washing facilities were explored, and hand washing facilities were observed in both intervention and control schools.

Results The "Hands up for Max!" resource was well received by the intervention schools, although some teachers made useful suggestions for improvements. Schools differed in the way they delivered the intervention and the number of elements of the resource package they used. Pupils in intervention schools recalled learning about the importance of hand washing in reducing the spread of infections and were able to describe, in detail, how to wash their hands properly. In the focus groups, pupils provided insight into reasons why they may not wash their hands, and what might help people wash their hands properly. Use of drawings in the focus groups facilitated discussion about what pupils liked and did not like about the facilities where they washed their hands. Results of the process evaluation were also used to inform development of questionnaires to obtain quantitative data from pupils and staff in all 178 schools participating in the trial.

Conclusion Information from the process evaluation will be useful in understanding any observed differences in quantitative outcomes related to absenteeism and knowledge, attitudes and behaviours related to hand washing, between intervention and control schools.
P25 ANTIBIOTIC PRESCRIBING IN IRISH CHILDREN RECEIVING FREE MEDICAL CARE

doi:10.1136/jech.2010.120477.25

${ }^{1} \mathrm{C}$ Keogh, ${ }^{1,2} \mathrm{U}$ Reulbach, ${ }^{1} \mathrm{~N}$ Motterlini, ${ }^{3} \mathrm{~K}$ Bennett, ${ }^{1} \mathrm{~T}$ Fahey. ${ }^{1}$ HRB Centre for Primary Care Research, Royal College of Surgeons Ireland, Dublin, Ireland; ${ }^{2}$ Public Health \& Primary Care, Trinity College Dublin, Dublin, Ireland; ${ }^{3}$ Pharmacology \& Therapeutics, Trinity College Dublin, Dublin, Ireland

Objective High rates of antibiotic prescribing are an increasing worldwide concern, in terms of increased antimicrobial resistance and associated burden to health services. However, there remains a lack of paediatric population-based pharmacoepidemiological research. The aim of the current study is to determine trends in systemic antibiotic prescribing in Irish children.

Design Retrospective analysis of routinely collected data.

Participants Data were obtained from the General Medical Services (GMS) claims database. This represents patients who are unable to pay for medical services and accounts for about one third of Irish children. Data were obtained from 2004 to 2008, for about 271000 children per year aged $\leq 15$.

Main Outcome Antibiotic prescribing behaviour in a paediatric population.

Results Prescribing rates of systemic antibiotics were compared across years, age $(0-4,5-11,12-15)$ and gender and are presented with CI and significance values (using negative binomial regression analysis). In 2004, 631/1000 population (95\% CI 628 to 634) received at least one antibiotic prescription compared to $578 / 1000$ ( $95 \%$ CI 575 to 581) in 2008. However, the overall trend across the 5 -year period did not show a significant change (IRR 0.99, 95\% CI 0.96 to $1.0, p=0.23)$. No significant difference was observed between males $(620 / 1000,95 \%$ CI 618 to 622$)$ and females $(631 / 1000,95 \%$ CI 629 to 633 ; IRR 1.02, 95\% CI 0.97 to $1.06, \mathrm{p}=0.46$ ). However, prevalence rates significantly decreased with age. Children in the 0-4 age group were significantly more likely to receive an antibiotic prescription $(827 / 1000,95 \%$ CI 824 to 829$)$, relative to the 5-11year old $(553 / 1000,95 \%$ CI 552 to 556$)$ and $12-15$ years $(489 / 1000$ $95 \%$ CI 487 to 492 ), with all $p<0.001$. The net ingredient cost of antibiotics increased from $€ 2.3$ million in 2004 to $€ 2.8$ million in 2008. The five most commonly prescribed drugs were as follows: amoxicillin (298/1000, 95\% CI 297 to 299), co-amoxiclav (296/1000, 95\% CI 295 to 296), cefaclor (135/1000, 95\% CI 134 to 136), clarithromycin (54/1000, 95\% CI 53 to 54 ) and phenoxymethylpenicillin (53/1000, 95\% CI 52 to 53). The overall rates observed here are significantly higher than those reported elsewhere in Europe. For example, the Netherlands (178/1000), Denmark (328/1000) and Scotland $(142 / 1000)$ consistently report low antibiotic prescribing rates. The choice of agents also differed between countries.

Conclusions Prescribing rates remained stable over the 5 -year period. Although the rates observed here are higher than European comparisons, these results should be considered in the context of the GMS population. Nevertheless, the results suggest the possible overuse of antibiotics within the GMS population and the potential benefit from interventions to reduce prescribing.

\section{Older people \\ P26 INTERVENTIONS TO IMPROVE PRESCRIBING QUALITY IN CARE HOMES: A SYSTEMATIC REVIEW}

doi:10.1136/jech.2010.120477.26

${ }^{1} \mathrm{M}$ Loganathan, ${ }^{1} \mathrm{~S}$ Singh, ${ }^{1}$ Alex Bottle, ${ }^{2} \mathrm{~B}$ D Franklin, ${ }^{1} \mathrm{~A}$ Majeed. ${ }^{1}$ Department of Primary Care and Public Health, Imperial College London, London, UK; ${ }^{2}$ Centre for Medication Safety and Service Quality, Imperial College Healthcare NHS Trust/The School of Pharmacy, University of London, London, UK

Introduction Prescribing in the elderly population is a complex process and the prevalence of inappropriate prescribing is high, with 